and intermediate GRACE risk classes $(50 \%$ vs $91.7 \%$ and $66.7 \%$ respectively, $p=0.03)$. Within the group who were treated invasively: early intervention within 2 hours or 24 hours was reserved for those with low and intermediate GRACE risk class, yet, there is no statistically significant difference in timing to intervention according to GRACE risk class $(p=0.95)$, see figure 1 . In-hospital acute heart failure was recorded in $8.7 \%$, cardiogenic shock was reported in $6.5 \%$, atrial fibrillation was documented in $2.2 \%$ while life threatening arrhythmias were recorded in $6.5 \%$, stroke or all in-hospital mortality occurred in none of young patient population.

Conclusion Current analysis explored that young patients who were presented with NSTE-ACS were mostly males and had smoking, hypertension and diabetes as the most common cardiovascular risk factors. They were mostly treated invasively, however, those with high GRACE risk class were treated more conservatively. Younger patients with NSTE-ACS had favorable in-hospital outcomes compared to what literature reported in older patients. Preventive programs should be the target measure to control cardiovascular risk factors to decrease incidence of IHD in younger population.

Conflict of Interest None

\section{IMPACT OF DIABETES ON MANAGEMENT OF PATIENTS WITH NON ST-ELEVATION ACUTE CORONARY SYNDROMES: A SPECIAL POPULATION WITH NO SPECIAL MANAGEMENT IN REAL WORLD PRACTICE}

Zainab Dakhil. Ibn Al Bitar Cardiac Centre, Al-kindy College Of Medicine/university of Baghdad, Baghdad, Iraq

\subsection{6/heartjnl-2021-BCS.68}

Background Atherosclerotic cardiovascular disease is the leading cause of morbidity and mortality in diabetic population, one of such cardiovascular presentations is Non ST-Elevation Acute Coronary Syndromes (NSTE-ACS). This fact coupled with that the Middle East is considered one of the global diabetes hot spots, yet there is lack of data from middle eastern countries including Iraq regarding the management of this population when presented as NSTE-ACS, so, we aimed to evaluate the impact of diabetes on management of patients with NSTE-ACS in terms of pharmacotherapy and adoption of invasive strategy in management.

Methods Hospitalised patients with NSTE-ACS were prospectively recruited in this study, baseline characteristics, laboratory results as well as in-hospital pharmacotherapy were all recorded. Mode of management (invasive vs conservative) and timing to intervention if done were also documented as well as in-hospital outcomes. GRACE risk score was calculated and documented by the investigator with subsequent classification into low, intermediate and high GRACE risk class. Patients were categorised into two groups: diabetics vs non-diabetics.

Results A total of 200 patients were included in this study, among whom $49 \%$ were diabetics, diabetic patients were older than non-diabetics $(60.5 \pm 11.4)$ vs $(57.1 \pm 11.9), p=0.04$, diabetics were females in $33.7 \%$ vs $25.5 \%, p=0.2$. Diabetics were more likely to have hypertension $(77.6 \%$ vs $61.8 \%$, $\mathrm{p}=0.01)$, IHD $(58.2 \%$ vs $38.2 \%, \mathrm{p}=0.005)$ and hyperlipidaemia $(28.6 \%$ vs $16.7 \%, \mathrm{p}=0.04)$. Troponin was positive in $(53.1 \%$ in diabetics vs $40.2 \%, \mathrm{p}=0.06)$, diabetics had higher

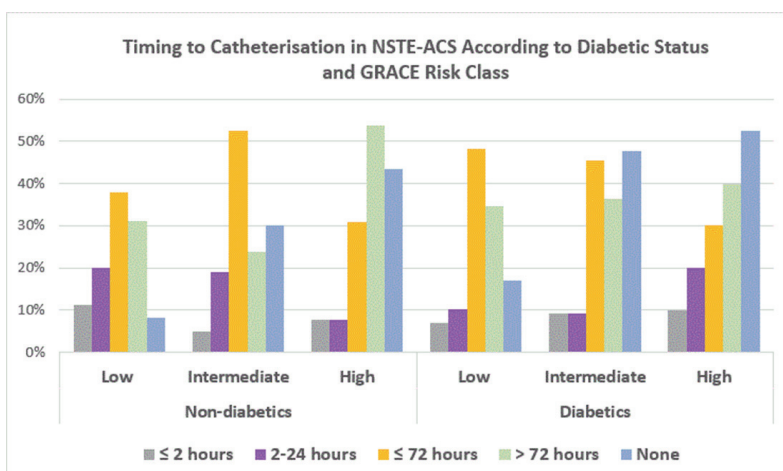

Abstract 68 Figure 1

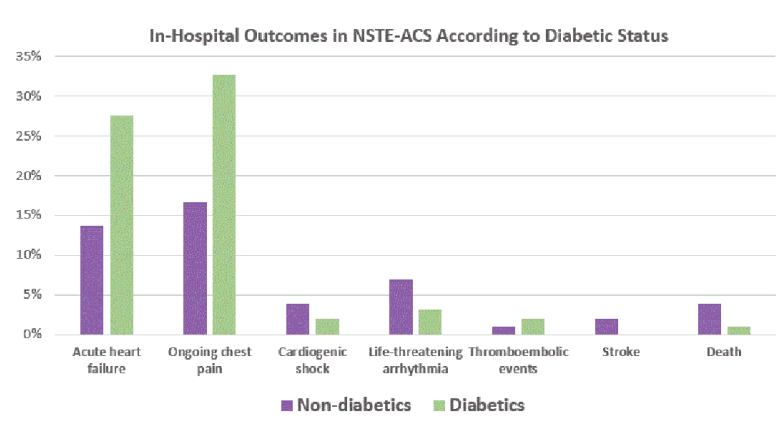

Abstract 68 Figure 2

blood glucose $(\mathrm{mg} / \mathrm{dl})(250.3 \pm 121.7$ vs $128.7 \pm 50.3, \mathrm{p}<0.001)$ and higher serum creatinine $(\mathrm{mg} / \mathrm{dl})(1.02 \pm 0.4$ vs $0.89 \pm 0.2$, $\mathrm{p}=0.005)$ compared to non-diabetics, while they had lower left ventricular ejection fraction\% $(51.3 \pm 11.6$ vs $56.2 \pm 11.8$, $\mathrm{p}<0.001)$. There were no statistical differences between the two groups in prescription of: aspirin, P2Y12 inhibitors, Bblockers, Calcium channel blockers, ACEI/ARBs, heparin, statins, mineralocorticoid receptor antagonist or loop diuretics, however, oral nitrate was used more in non-diabetics compared with diabetics $(70.6 \%$ vs $51 \%, \mathrm{p}=0.005)$. Diabetics were presented at higher GRACE risk class (GRACE score $>140$ in $42.9 \%$ vs $35.4 \%, p=0.002$ ), yet they were less likely to be referred for catheterisation compared with nondiabetic counterparts $(61.2 \%$ vs $77.5 \%, \mathrm{p}=0.01)$, however, for those who were referred for catheterisation, timing to catheterisation was not statistically significant between the two groups, see figure 1. Diabetics were more likely to develop acute heart failure (AHF) and ongoing ischaemic chest pain during hospitalization $(27.6 \%$ vs $13.7 \%, \mathrm{p}=0.01)$ and $(32.7 \%$ vs $16.7 \%, \mathrm{p}=0.009$ ) respectively, however, no significant differences were observed between the two groups in developing life-threatening arrhythmias, cardiogenic shock, stroke or death, see figure 2 .

Conclusion Despite that international guidelines in management of NSTE-ACS have labelled patients with diabetes and NSTE-ACS as special population considering their high risk and recommended early revascularization strategy, there is underutilization of invasive strategy in managing this population, yet timing to intervention was not impacted by diabetic status. Further work is needed to clarify reasons of underuse of invasive strategy in diabetic population in order to improve adherence to guidelines.

Conflict of Interest None 